\title{
Kinetic behaviors of Inulin hydrolysis by inulinase from Kluyveromyces
}

\author{
Zhao Zhao ${ }^{\text {a }}$, Jiaoyu Shi ${ }^{\mathrm{b}}$ and Zhiping Zhao ${ }^{\mathrm{c*}}$
}

\author{
School of Chemical Engineering and the Environment, Beijing Institute of Technology, Beijing \\ 100081, China. \\ azhaozha0851023@163.com, bjiaoyushicn@foxmail.com, ${ }^{\text {c* } z h a o z p @ b i t . e d u . c n ~}$ \\ Corresponding Author: Zhiping Zhao
}

Keywords: inulin, inulinase, hydrolysis, kinetic models.

\begin{abstract}
Enzyme hydrolysis kinetic model of inulin by inulinase from Kluyveromyces lactis was established. The corresponding Michaelis-Menten equation model constants were found to be related to temperature through the Arrhenius model. The kinetic model was validated by experimental data and also be utilized to predict the hydrolysis reaction rates of the inulin for substrate concentrations ranging between 2 and $200 \mathrm{~g} / \mathrm{L}$ and a fairly large reaction temperature range (35-55 $\left.{ }^{\circ} \mathrm{C}\right)$. Furthermore, this kinetic model can provide a scientific and convenient way to choose the optimized hydrolysis conditions for the production of fermentable sugar for ethanol production.
\end{abstract}

\section{Introduction}

Over the last decades, energy shortages and global climate change, together with the increasing dependence on petroleum resources, have reinforced the need of using renewable resources as an alternative to fossil fuels [1]. Jerusalem artichoke has been found to be one of the most biomass resources for biofuel production. Inulin in Jerusalem artichoke is a fructose polymer which mainly consists of $\beta-(2 \rightarrow 1)$ fructosyl fructose units with normally a glucose unit at the reducing end. Fermentable sugar produced from inulin is the raw material for bioethanol production. And enzyme hydrolysis is a green and efficient way to produce fermentable sugar [2].

Kinetics analysis and further modeling studies of hydrolysis are valuable in the processing of Jerusalem artichoke to fermentable sugars. Enzymatic hydrolysis of polysaccharide biomass depends on many factors: physical properties of the substrate (crystallinity of cellulose, particle size, composition, degree of polymerization, etc.), enzyme characteristics (origin, synergy, etc.) and mass transfer (substrate adsorption, diffusion rate, etc.) [3-5]. Santos et al. [6] reported a novel method to establish the most suitable operational temperature for an enzyme reactor design which was based on mathematical modeling of the thermal stability and activity of the enzyme. Peri et al [7] investigated the kinetics of the cellulose enzyme complex as observed with hydrolysis of noncrystalline cellulose (NCC). Establishing a comprehensive and concise model which spans a relative wide substrate concentration is an essential procedure for the hydrolysis of inulin.

\section{Theoretical}

The inulin hydrolysis process the stoichiometric relationship can be described as:

(Fructan) $)_{\mathrm{n}}+n$-1water $\stackrel{\text { exo-inulinase }}{\longrightarrow} n$ (Fructose) + Glucose

According to the equation (1), the kinetics will appear first-order (i.e. pseudo-first-order reaction). The exo-inulinase catalyzed reaction mechanism can be described as bellows:

$$
E+S \stackrel{k_{1}}{\longrightarrow} E S \stackrel{k_{2}}{\longrightarrow} E+P
$$

In this reaction mechanism, the first step, binding of substrates, would be rapid compared to the subsequent chemical steps that converted substrate to product (in rapid equilibrium), so that at saturation by the substrate the rate would be equal to the microscopic rate constant $k_{2}$. The hydrolysis of inulin exhibited a Michaelis-Menten kinetics. The kinetic parameters were first estimated by linear 
regression using the Lineweaver-Burk plot, which is deduced from the linearsation of the Michaelis-Menten equation:

$$
V_{0}=\frac{V_{\max } S_{0}}{K_{\mathrm{m}}+S_{0}}
$$

where $S_{0}$ is the concentration of inulin, Michaelis constant $K_{\mathrm{m}}$ is the substrate concentration at which the reaction rate is half of $V_{\max }$, and $V_{\max }$ is the maximum reaction rate.

The linear transform of the Michaelis-Menten equation devised by

$$
\frac{1}{V_{0}}=\frac{1}{V_{\max }}+\frac{K_{\mathrm{m}}}{V_{\max }} \cdot \frac{1}{S_{0}}
$$

where Michaelis constant $K_{\mathrm{m}}$ is the substrate concentration at which the reaction rate is half of $V_{\mathrm{m}}$, and $V_{\mathrm{m}}$ is the maximum reaction rate. In particular, the value of maximum reaction rate $\left(V_{\max }\right)$ can be described as:

$$
V_{\text {max }}=k_{2} e_{0}
$$

where $k_{2}$ is maximum number of substrate molecules converted to product per enzyme molecule per second, $e_{0}$ is the initial enzyme activity $(\mathrm{U} / \mathrm{ml})$. The dependence of the kinetic parameters $\left(K_{\mathrm{m}}\right.$ and $k_{2}$ ) on reaction temperature $T$ was established according to the Arrhenius equation (4) for enzymatic reactions:

$$
K=A \cdot \exp \left(\frac{E_{\mathrm{a}}}{R \mathrm{~T}}\right)
$$

Taking the natural logarithms gives:

$$
\ln K=\ln A-\frac{E_{\mathrm{a}}}{R \mathrm{~T}}
$$

where $R$ is gas constant, $T$ is the reaction temperature, and $E_{\mathrm{a}}$ is apparent activation energy. From the Lineweaver-Burk plot $\left(1 / V_{0}\right.$ versus $\left.1 / S_{0}\right)$ and classical Arrhenius plot by experimental data, the kinetic parameters will be obtained..

\section{Materials and methods}

\subsection{Materials.}

Inulin (derived from Jerusalem artichoke purchased from Gansu Likang nutrition and Food Co, Ltd.). The Kluyveromyces lactis MW270-7B strain was kindly provided by associate professor Jianping Liu (State Key Laboratory of Genetic Engineering, Institute of Genetics, School of Life Sciences, Fudan University); Chemicals were purchased from Oxoid Ltd., Basingstoke, Hampshire, England.

\subsection{Microorganism and enzyme production.}

The liquid media for inulinase production contains $1 \%$ yeast extract, $2 \%$ glucose and $2 \%$ peptone. A $500 \mathrm{ml}$ Erlenmeyer flask with $200 \mathrm{ml}$ medium was inoculated with $4 \mathrm{ml}$ seed culture medium and incubated in a rotator shaker at $30^{\circ} \mathrm{C}$ with the stirring speed of $170 \mathrm{rpm}$ for $120 \mathrm{~h}$.

\subsection{Initial velocity of hydrolysis tests.}

Initial velocity tests were used to determine the kinetic rate equation. Inulin at different concentrations $\left(2,3,5,10,20,50,100,200\right.$ g. $\left.\mathrm{L}^{-1}\right)$ were hydrolysis in a 50 ml flask with a reaction volume of $20 \mathrm{ml}$, the original enzyme concentration $0.5 \mathrm{U} / \mathrm{ml}$. reactions were performed in $50 \mathrm{mM}$ sodium acetate buffer at different temperatures. The range of temperature values considered was $35-55^{\circ} \mathrm{C}$. The total amount of reducing sugars released was detected by the 3, 5-dinitrosalicylic acid (DNS) method.

\subsection{Hydrolysis of inulin.}

Original enzyme concentration for inulin hydrolysis is $0.5 \mathrm{U} / \mathrm{ml}$. Reactions were performed in 50 mM sodium acetate buffer, $\mathrm{pH} 4.6$ at different temperatures. 


\section{Results and discussion}

\subsection{Kinetic parameters and models.}

The data of initial velocity test for inulin hydrolysis were plotted according Eq. (4). As shown in Fig. 1, the kinetics of hydrolysis of inulin followed Michaelis-Menten kinetic behavior under the range of substrate concentrations this work studied. Fig. 2 shows first order kinetics was observed in the whole concentration range. When observing the kinetic behaviors under different temperatures, it is clear that the initial velocity is much higher in $55^{\circ} \mathrm{C}$. This can be attributed to a higher enzyme activity under a relative higher temperature.

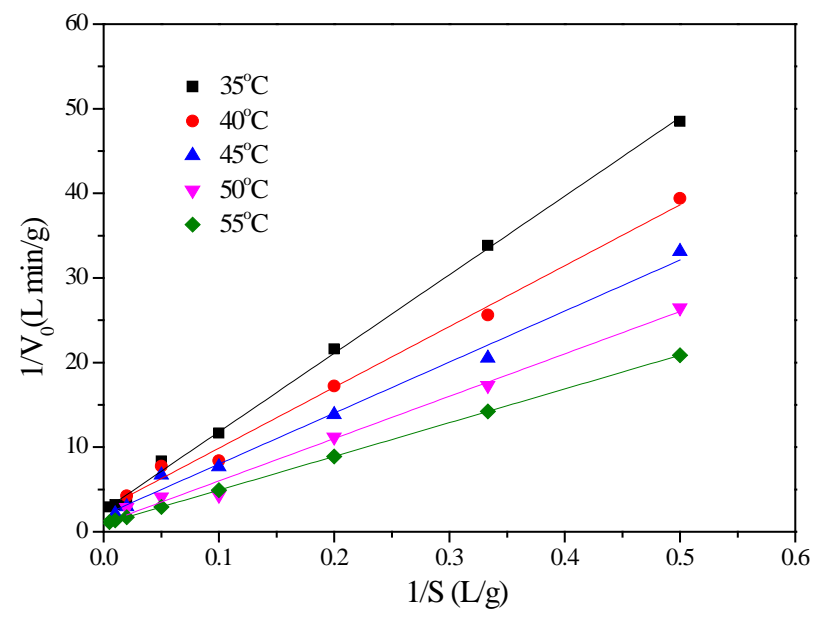

Fig. 1 Lineweaver-Burk interpolation of inulin hydrolysis at different temperatures.

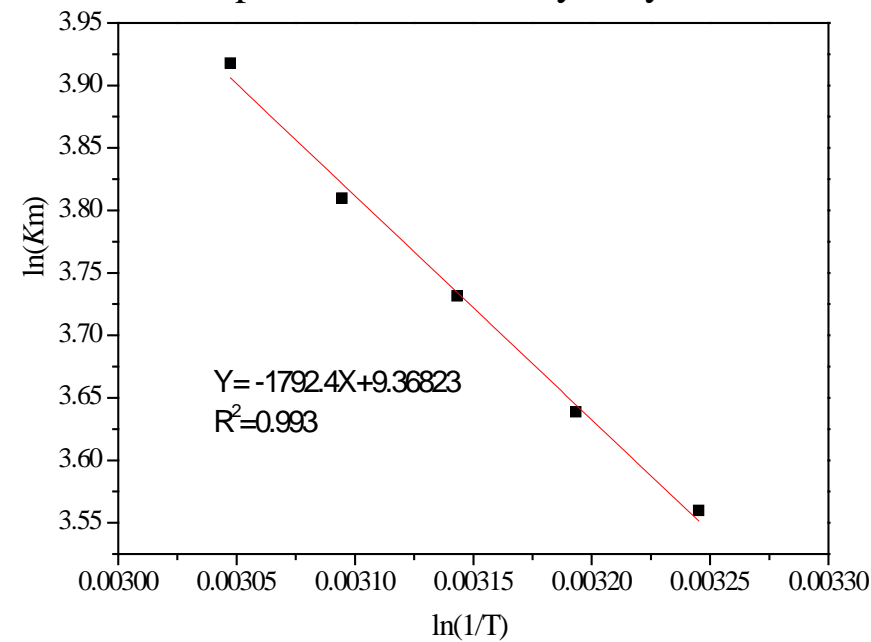

Fig. 2 Arrhenius plot of $\mathrm{Km}$ in hydrolysis of inulin

\section{Models Validation}

The values of $K \mathrm{~m}$ and $k_{2}$ obtained from (5) and (7) were used to determine the reaction rate $V$ according to Michaelis-Menten model with all inulin concentrations and at all temperatures. The resultant equations were:

$$
V(S, T)=\frac{\exp (16.58-7317 / T) e_{0} S}{\exp (9.368-1792 / T)+S}
$$

Validation of the proposed models was performed by comparing model predictions to experimental data. To valiated the reliability of the proposed models mentioned above, the simulation results by the model under different operation conditions were compared with the experimental data at the same conditions. The compared results between the prediction data and the experimental data for inulin hydrolysis was presented in Fig. 3. 


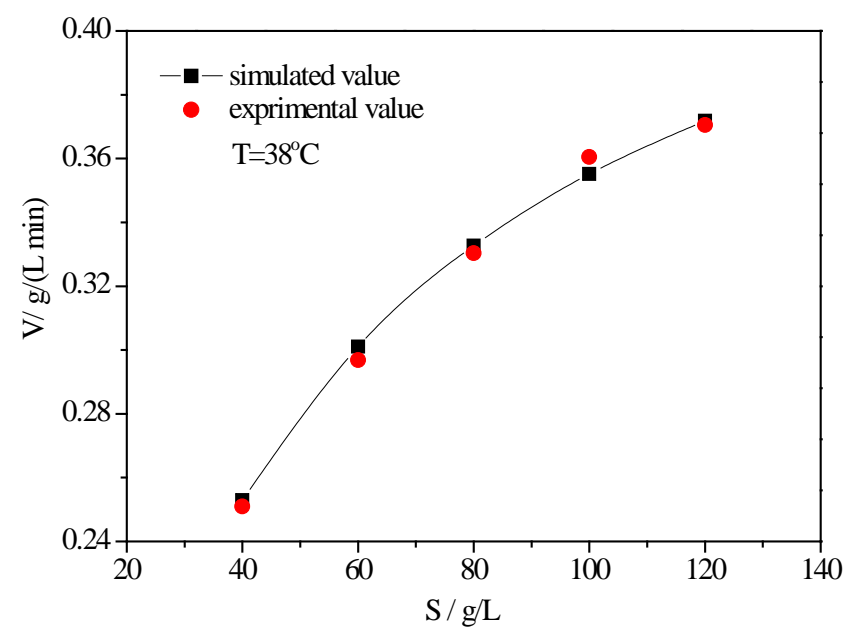

Figure 3 Model validations for hydrolysis of inulin

The different values of the experimental data and the simulation ones were mainly attributed to that the higher experimental error at low concentration of the substrate. When the concentration increases, the reaction system becomes increasingly viscous because of the existence of polysaccharide and the other insoluble substance. In fact, to improve the prediction accuracy of the hydrolysis models requires a high purity form of biomass substrate and a good mass transfer condition to minimize the error in the process of estimating kinetic parameters.

\section{Summary}

The experiments on the hydrolysis of inulin by inulinase secreted by the strain of Kluyveromyces lactis, which were carried out over a wide range of temperatures at $\mathrm{pH}$ 4.6. Based upon the classical Michaelis-Menten kinetic model integrated with the Arrhenius equations, the kinetic model for inulin hydrolysis was established. Agreement between experimental and theoretical data especially for a high concentration of substrate also demonstrated that the models have very practical significance in the production of high concentration fructose syrup during the bioethanol preparation.

\section{Acknowledgements}

The authors would like to thank the support of the National Natural Science Foundation of China (No. 21276024). We also appreciate associate professor Jianping Liu (State Key Laboratory of Genetic Engineering, Institute of Genetics, School of Life Sciences, Fudan University) for kindly providing us the KLuyveromyces lactis MW270-7B recombinant strain.

\section{References}

[1]. M. E. Himmel, S.-Y. Ding, D. K. Johnson, W. S. Adney, et al. Biomass Recalcitrance: Engineering Plants and Enzymes for Biofuels Production. Science, Vol. 315 (2007) No.9, p. 804-807.

[2]. S. E. Fleming, J. W. D. GrootWassink, E. D. Murray, et al. Preparation of high - fructose syrup from the tubers of the Jerusalem artichoke (Helianthus tuberosus L.). C R C Critical Reviews in Food Science and Nutrition. Vol. 12 (1979), p. 1-28.

[3]. E. Ricca, V. Calabrò, S. Curcio, et al. Optimization of inulin hydrolysis by inulinase accounting for enzyme time and temperature dependent deactivation. Biochemical Engineering Journal. Vol. 48 (2009), p. 81-86.

[4]. E. Ricca, V. Calabrò, S. Curcio et al. The state of the art in the production of fructose from inulin enzymatic hydrolysis. Critical Reviews in Biotechnology. Vol. 27 (2007), p. 129-145.

[5]. P. Carniti, P. L. Beltrame, D. Guardione et al. Hydrolysis of inulin: A kinetic study of the reaction catalyzed by an inulinase from Aspergillus ficuum. Biotechnology and Bioengineering. Vol. 37 
(1991), p. 575-579.

[6]. A. M. P. Santos, M. G. Oliveira, F. Maugeri, et al. Modelling thermal stability and activity of free and immobilized enzymes as a novel tool for enzyme reactor design. Bioresource Technology, Vol. 98 (2007) No.11, p. 3142-3148.

[7]. S. Peri, S. Karra, Y. Y. Lee, et al. Modeling intrinsic kinetics of enzymatic cellulose hydrolysis. Biotechnology Progress, Vol. 23 (2007), pp. 626-637. 